\title{
Auditory short-term memory in the budgerigar (Melopsittacus undulatus)
}

\author{
JANE D. DOWNING, KAZUO OKANOYA, and ROBERT J. DOOLING \\ University of Maryland, College Park, Maryland
}

\begin{abstract}
We trained two budgerigars (Melopsittacus undulatus) with operant techniques in a delayed matching-to-sample task using pairs of acoustic stimuli. These stimuli included simple pure tones, complex, species-specific vocalizations, and tone-vocalization combinations. The birds were then tested with different retention intervals. The budgerigars' short-term memory was similar for complex, species-specific vocalizations and for simple pure tones. By contrast, they showed significantly better short-term memory when tested with two sounds drawn from different acoustic categories.
\end{abstract}

The budgerigar, or parakeet (Melopsittacus undulatus), is a small Australian parrot with a complex vocal repertoire, some of which develops through learning (Brockway, 1964; Dooling, 1986; Dooling, Gephart, Price, McHale, \& Brauth, 1987). Operant conditioning experiments have shown that budgerigars can learn and remember for long periods of time an auditory discrimination involving species-specific calls (Park \& Dooling, 1985). Additional tests reveal that budgerigars can also maintain discrimination among a large set of contact calls when those calls are severely degraded (Park \& Dooling, 1986). Psychophysical experiments confirm that budgerigars are unusually sensitive to spectral changes occurring in the frequency region of $2-4 \mathrm{kHz}$ (Dooling, 1982). In aggregate, these results suggest that budgerigars may code species-specific vocal signals using a specialized process involving sensory, memory, and attentional components. The present experiment measures auditory short-term memory (STM) of budgerigars.

Most animal work on STM has involved visual stimuli (Grant, 1981; Roberts \& Grant, 1976), but several recent studies have used auditory stimuli. These studies involved delayed matching-to-sample (DMTS) procedures, combined with auditory-auditory (A-A) discrimination tasks in which the animal was required to distinguish between two different sounds. Because the matching task has proved unusually difficult for animals, the availability of comparative literature is limited (Shyan, Wright, Cook, \& Jitsumori, 1987). As one example, D'Amato and Colombo (1985) reported that only four out of eight monkeys were able to learn the A-A matching-to-sample task. The monkeys that learned the task were able to retain the sample stimulus over an interval of roughly $3 \mathrm{sec}$.

This work was supported by NIH Grants NS19006 and HD00512 to R. Dooling. This article is based on an Honor's Thesis submitted by Jane Downing in partial fulfillment of a Bachelor of Science Degree of Psychology from the University of Maryland. Correspondence concerning this article should be addressed to Robert J. Dooling, Department of Psychology, University of Maryland, College Park, MD 20742.
As far as we know, rats and pigeons have yet to be tested for short-term memory using A-A discriminations, but they have been tested for their ability to retain crossmodality (visual-auditory) discriminations (Cohen, Escott, \& Ricciardi, 1984; Kraemer \& Roberts, 1984). In these studies, the ability of animals to match modalities was examined. The animals were trained to respond to two successive visual stimuli (V-V) or two successive auditory stimuli (A-A) and to withhold responses to either A-V or V-A stimulus combinations. Maximum retention intervals for STM ranged between a minimum of roughly $1 \mathrm{sec}$ for the rat to a maximum of roughly $5 \mathrm{sec}$ for the pigeon.

The dearth of auditory STM studies in animals also means that there have been no studies using natural vocalizations (but see D'Amato \& Salmon, 1984; Herman, Richards, \& Wolz, 1984). This is remarkable, because there are good reasons for thinking that animals might excel at such a task. Humans, for example, show a significant increase in auditory STM in tests with meaningful speech stimuli as compared with tests using nonspeech stimuli (see, as examples, Dowling \& Braun, 1957; Ellis, Parente, \& Shumante, 1974; Peterson, Peterson, \& Miller, 1961).

The present experiment examined whether STM for vocal signals in budgerigars is significantly different from that observed for simple acoustic stimuli. Characterizing the nature of auditory STM in budgerigars is especially interesting since, like many song birds (Kroodsma \& Miller, 1982), some of the species-specific vocalizations of the budgerigar develop through learning (Dooling, Gephart, et al., 1987).

\section{METHOD}

\section{Subjects}

We used two male budgerigars (B1 and B2) in the present experiment. Both subjects had previous experience on operant conditioning tasks involving auditory stimuli. The animals were food deprived for approximately $12 \mathrm{~h}$ before the testing sessions (Park, Okanoya, \& Dooling, 1985). Standard mixed parakeet seed was 
used as the reinforcer during the experimental sessions, and was also available during free-feeding times. The birds were housed in aviaries at the University of Maryland.

\section{Apparatus}

The apparatus used for training and testing has been described previously (Park \& Dooling, 1985; Park et al., 1985). The birds were tested in a small wire cage mounted within a sound-attenuation chamber. A standard pigeon grain hopper was used for reinforcement delivery. The response keys (observation key and report key) were constructed from microswitches and LEDs, and were mounted just above the feeder opening. An IBM Personal Computer AT controlled the operant apparatus, arranged contingencies, presented stimuli, and recorded data. Tone bursts and natural contact calls were stored digitally in the firmware memory of the computer. Both tones were $150 \mathrm{msec}$ in duration-the average duration of contact calls in this species (Dooling, 1986). The two contact calls (Cl and C2) used in these experiments were $145 \mathrm{msec}$ and $157 \mathrm{msec}$ in duration, respectively. All stimuli were presented at a level of about $76 \mathrm{dBA}$ SPL in the chamber through a loudspeaker mounted $14 \mathrm{~cm}$ directly above the bird's head.

\section{Procedure}

Training. We trained the birds in daily sessions consisting of roughly 180 trials. A response on the observation key initiated a trial. Following this observation response, two stimuli were presented, separated by a retention interval (RI) of $250 \mathrm{msec}$. For any two stimuli ( $A$ and $B$ ), the possible combinations of presentations were A-A, B-B, A-B, and B-A. In each session, all combinations of the stimulus pairs were presented an equal number of times in random order. If the stimulus pair was A-B or B-A (GO stimuli), a peck on the report key within 2 sec was reinforced by a 2-sec access to feed. Failure to respond during the 2-sec response period led to a 1-sec intertrial interval (ITI), during which the LED on the observation key was extinguished, followed by the initiation of another trial sequence. If the stimulus pair was A-A or B-B (NOGO stimuli), a peck on the report key within $2 \mathrm{sec}$ resulted in a time-out period of $20 \mathrm{sec}$, during which the chamber light was extinguished. No response during the 2 -sec response period led to a 1-sec ITI, again followed by the initiation of another trial sequence. The ITI was nominally $1 \mathrm{sec}$. However, the minimum period between presentations of stimulus pairs was $3 \mathrm{sec}$ (i.e., a 2-sec response period plus a $1-\mathrm{sec}$ ITI), whereas the maximum period was $23 \mathrm{sec}$, which occurred following an incorrect response (i.e., a 2-sec response period, a 1-sec ITI, plus a 20-sec time-out period).

The training portion of the experiment ended when the birds reached a correct-response rate of $85 \%$ for both same and different trials for three consecutive sessions.

Testing. The testing procedures were the same as the training procedures except for the length of the RI. The RI for the pair of stimuli presented was chosen randomly from $125,250,500,1,000$, or $2,000 \mathrm{msec}$. The trials were presented in blocks of ten, with each RI randomly presented twice. Testing continued as long as the average of the correct-response rate from the 125- and 250-msec RIs remained at $85 \%$ with neither falling below $80 \%$. The testing period for one set of stimuli ended with the completion of five consecutive sessions that met the designated criteria. Perfect performance occurred at $100 \%$ correct, while chance performance occurred at $50 \%$ correct. The threshold RI from each testing session that met the criteria was defined as the length of the delay corresponding to a correct-response rate of $75 \%$.

The experiment involved three conditions, each of which used only one pair of stimuli. The first condition involved training and testing on two pure-tone bursts of 2000 and $2860 \mathrm{~Hz}$. These stimuli fall within the budgerigar's most sensitive hearing region as determined by psychophysical studies (Dooling, 1982). The second condition involved training and testing birds with two budgerigar con- tact calls ( $\mathrm{Cl}$ and $\mathrm{C} 2$ ). These calls were chosen from pilot data showing these calls sound very different to budgerigars (Dooling, Park, Brown, Okanoya, \& Soli, 1987). Sonograms of the budgerigar contact calls used as stimuli are shown in Figure 1. The third condition involved a $2860-\mathrm{Hz}$ pure tone and one of the previously used contact calls (C2) as the stimulus pair.

Following the completion of testing under each condition, both birds were retested with the original pair of tonal stimuli to test for practice effects. As an additional check for practice effects, one bird was retested with the pair of contact-call stimuli.

\section{RESULTS}

One result of the present experiments is that the acquisition of the A-A discrimination task was very difficult for budgerigars. Budgerigars B1 and B2 required 24 and 84 sessions, respectively, to master the task for the pair of tones. These same birds required 27 (B1) and 105 (B2) sessions to learn the task involving the pair of contact calls.

Results from both subjects are shown as retention gradients in Figure 2. Retention gradients for both stimulus sets were obtained by plotting the mean correct-response percent at each RI. The birds' performance for the RI of $125 \mathrm{msec}$ was generally better than $90 \%$, whereas at the longest RI of $2,000 \mathrm{msec}$, performance approached chance levels $(50 \%)$. For tests involving stimuli drawn from the same acoustic category (a pair of tones or a pair of calls), the threshold RI (75\% correct-response point) fell between 430 and $560 \mathrm{msec}$. When the pair of stimuli was drawn from different acoustic categories (i.e., a call paired with a tone), the threshold RI fell between 690 and $850 \mathrm{msec}$.

A two-way analysis of variance (ANOVA) (2 subjects $\times 3$ test conditions, with unequal sessions per cell) was used to test the effect of stimulus categories and individual differences on threshold RIs. A total of $\mathbf{4 5}$ sessions was available for analysis. Since neither bird showed significant practice effects for tones or for calls, we included

\section{BUDGERIGAR CONTACT CALLS}

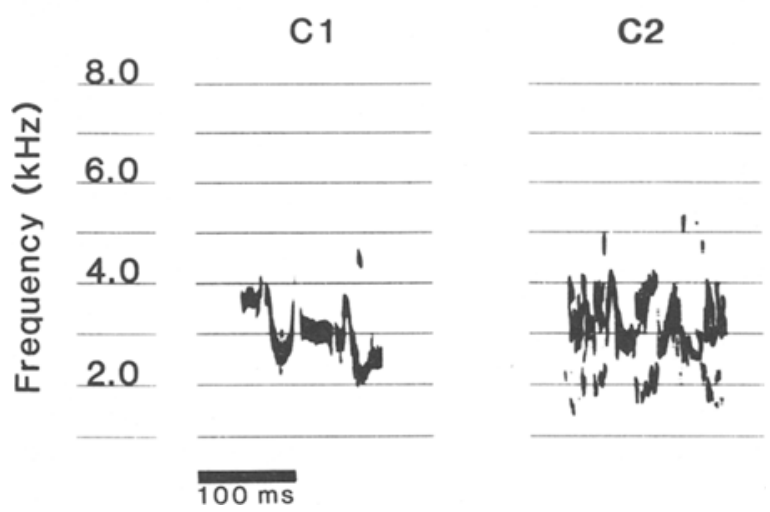

Figure 1. Sonograms of the two budgerigar contact calls used as test stimuli. Frequency is on the ordinate in kilohertz. The time marker is $\mathbf{1 0 0}$ msec. 


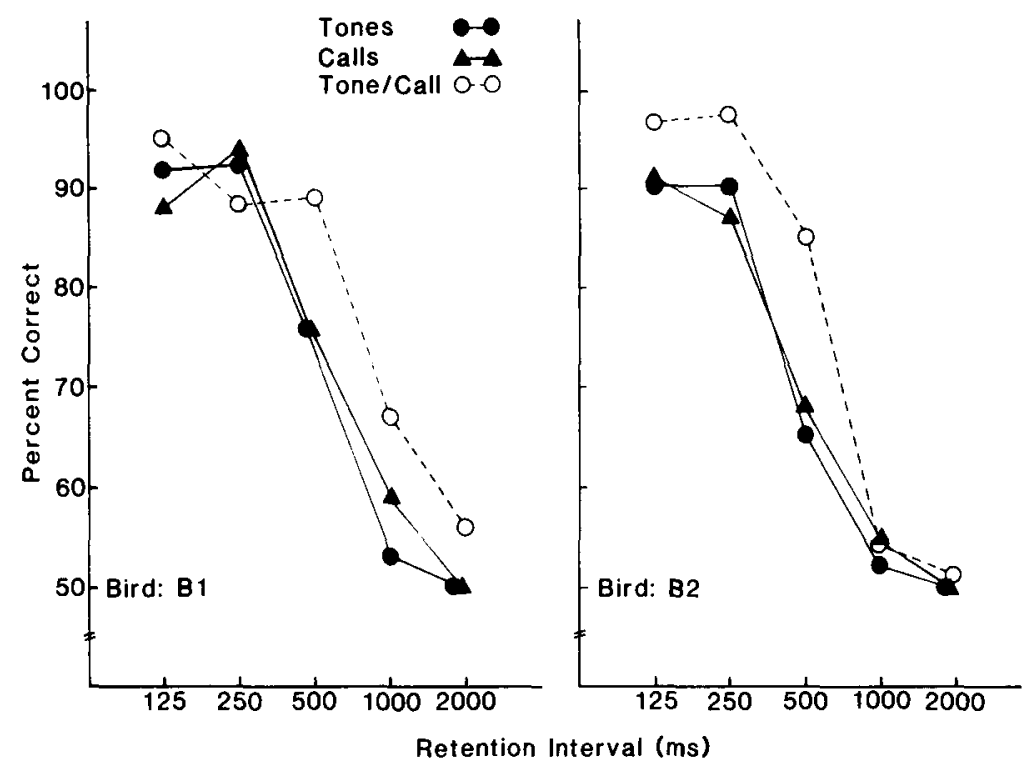

Figure 2. Performance of the two budgerigars on three sets of stimuli. Performance on two sets of within-category stimuli (a pair of tones and a pair of budgerigar contact calls) is shown by solid lines and closed symbols. Performance on the set of betweencategory stimuli (a tone and a budgerigar contact call) is shown by a dashed line and open symbols.

the data collected from the retesting sessions in this analysis. The effect of stimulus type on threshold RIs was significant $[F(2,39)=22.37, p<.01]$.

The third condition resulted in much longer RIs for each subject. Thus, it is likely that this condition was largely responsible for the significant effect across type of stimuli. To test this hypothesis, we performed several contrast analyses with a two-way ANOVA. First, the condition using a pair of tones and the condition using a pair of calls were contrasted with the condition using a tone-call combination. This contrast was significant $[F(1,39)=43.78$, $p<.01]$. A second analysis contrasted the condition using a pair of tones with the condition using a pair of calls. This analysis showed no significant contrast $[F(1,39)=$ $.105, p>.50$ ]. We conclude that the threshold RI found in the third condition (tone-call) was significantly longer than that for the first and second conditions (tone-tone and call-call, respectively).

\section{DISCUSSION}

These results show that budgerigars can be trained, albeit with some difficulty, to make auditory-auditory discriminations using a go/no-go procedure. Furthermore, the pattern of decay in auditory STM in budgerigars is similar to that observed for other animals tested on both auditory and visual STM tasks (see, as examples, D'Amato \& Colombo, 1985; D'Amato \& Salman, 1984; Herman, 1980; Herman \& Thompson, 1982; Kojima, 1985; Kraemer \& Roberts, 1984). The absolute time span of budgerigars' auditory STM, however, seems short compared with that reported for other animals. The RI for monkeys (Kojima, 1985), dolphins (Herman, 1980;
Herman \& Thompson, 1982), and humans (Crowder, 1982; Pisoni, 1973), when tested under similar conditions, is considerably longer than that reported here for budgerigars.

Part of the rationale for testing budgerigars on natural vocalizations comes from work testing humans on speech that showed that phonetic boundaries and stimulus meaning could have a strong effect on STM (Crowder, 1982; Dowling \& Braun, 1957; Pisoni, 1973). Since contact calls are natural, biologically relevant acoustic signals for which budgerigars have extensive experience, we expected that auditory short-term memory would be longer for contact calls than for pure tones. Contrary to our expectations, the results show there was no significant difference in STM for the tone pair compared to the contactcall pair.

The fact that short-term memory in budgerigars on "between-category" comparisons (i.e., a tone paired with a call) was significantly better than performance on "within-category" comparisons (i.e., a tone paired with a tone or a call paired with a call) proves that these procedures are sensitive enough to show the effect of different stimuli. The straightforward conclusion, then, is simply that the difference between the two pure tones and between the two contact calls is in some way less salient than the difference between the contact calls and the pure tones.

Modest as it appears, this result has considerable significance for the larger issue of whether organisms are "specialized" for the perception of species-specific vocal signals (Dooling, 1986). "Specialization" refers to evidence that a species is adapted to process speciesspecific acoustic signals more efficiently than other 
sounds. In the budgerigar, for example, there is already strong evidence for natural perceptual categories that correspond to functional and acoustic classes of vocal signals (Dooling, Park, et al., 1987). The results from the present study showing a similar STM for tones and calls in budgerigars suggest that specialized processes are not involved. In other words, the perception of contact calls and the perception of simple, nonmeaningful stimuli might involve similar auditory and memory processes in budgerigars.

Another possibility, and an important caveat, is that context might play a critical role. The "meaning" of contact calls for budgerigars might be somewhat distorted in an operant conditioning environment-an environment quite different from the natural communicative context in which contact calls are normally used by budgerigars. This natural context might turn out to be crucial for providing evidence for short-term memory specializations related to vocal signals in animals. Short-term memory specializations for species-specific vocal signals might, if they exist at all, only emerge under natural communicative circumstances.

\section{REFERENCES}

BrockWAY, B. F. (1964). Ethological studies of the budgerigar (Melop sittacus undulatus): Nonreproductive behavior. Behavior, 22, 192-222.

COHEN, J. S., Escott, M., \&icciardi, P. (1984). The role of reinforcement symmetry and stimulus modality in successive delayed matching to sample in the rat. Canadian Journal of Psychology, 38, 63-79.

Crowder, R. G. (1982). Decay of auditory memory in vowel discrimination. Journal of Experimental Psychology: Learning, Memory, \& Cognition, 8, 153-162.

D'Amato, M. R., \& Colombo, M. (1985). Auditory matching-to-sample in monkeys (Cebus apella). Animal Leaming \& Behavior, 13, 375-382.

D'Amato, M. R., \&alman, D. P. (1984). Processing and retention of complex auditory stimuli in monkeys. Canadian Journal of Psychology, 38, 237-255.

Dooling, R. (1982). Auditory perception in birds. In D. E. Kroodsma \& E. H. Miller (Eds.), Acoustic communication in birds (Vol. 1, pp. 95-130). New York: Academic Press.

Dooling, R. (1986). Perception of vocal signals by the budgerigar (Melopsittacus undulatus). Experimental Biology, 45, 195-218.

Dooling, R. J., Gephart, B. F., Price, P. H., Mchale, C., \& Brauth, S. E. (1987). Effects of deafening on the contact calls of the budgerigar (Melopsittacus undulatus). Animal Behaviour, 35, 1264-1266.
Dooling, R., Park, T., Brown, S., Okanoya, K., Soli, S. D. (1987). Perceptual organization of acoustic stimuli in budgerigars (Melopsittacus undulatus): II. Vocal signals. Journal of Comparative Psychology, 101, 367-381.

Dowling, R. M., \& Braun, H. W. (1957). Retention and meaningfulness of material. Joumal of Experimental Psychology, 54, 213-217.

Eluis, H. C., Parente, F. J., \& Shumante, E. C. (1974). Meaningfulness, perceptual grouping, and organization in recognition memory. Journal of Experimental Psychology, 102, 308-313.

GRANT, D. S. (1981). Short-term memory in the pigeon. In N. E. Spear \& R. R. Miller (Eds.), Information processing in animals: Memory mechanisms (pp. 227-255). Hillsdale, NJ: Erlbaum.

Herman, L. M. (1980). Cognitive characteristics of dolphins. In L. M. Herman (Ed.), Cetacean behavior: Mechanisms and functions (pp. 363-429). New York: Wiley Interscience Press.

Herman, L. M., Richards, D. G., Wolz, J. P. (1984). Comprehension of sentences by bottlenosed dolphins. Cognition, 16, 129-219.

Herman, L. M., Thompson, R. K. R. (1982). Symbolic, identity, and probe delayed matching of sounds by the bottlenosed dolphin. Animal Learning \& Behavior, 10, 22-34.

KonmA, S. (1985). Auditory short-term memory in the Japanese monkey. International Journal of Neuroscience, 25, 255-262.

Kraemer, P. J., \& Roberts, W. A. (1984). Short-term memory for visual and auditory stimuli in pigeons. Animal Learning \& Behavior, 12, 275-284

Kroodsma, D. E., Miller, E. H. (1982). Acoustic communication in birds (Vol. 1). New York: Academic Press.

Park, T. J., \& Dooulng, R. J. (1985). Perception of species-specific contact calls by the budgerigar (Melopsittacus undulatus). Journal of Comparative Psychology, 99, 391-402.

Park, T. J., \& Dooling, R. J. (1986). Perception of degraded vocalizations by budgerigars (Melopsittacus undulatus). Animal Learning \& Behavior, 14, 359-364.

Park, T., Okanoya, K., \& Dooling, R. (1985). Operant conditioning of small birds for acoustic discrimination. Journal of Ethology, $3,5-9$.

Peterson, L. R., Peterson, M. J., \& Miller, A. (1961). Short-term memory retention and meaningfulness. Canadian Joumal of Psychology, 15, 143-147.

Pisoni, D. B. (1973). Auditory and phonetic memory codes in the discrimination of consonants and vowels. Perceptual Psychophysiology, 13, 253-260.

RoberTs, W. A., \& Grant, D. S. (1976). Studies of short-term memory in the pigeon using the delayed matching-to-sample procedure. In $\mathrm{D}$. $\mathrm{L}$. Medin, W. A. Roberts, \& R. T. Davis (Eds.), Processes of animal memory (pp. 79-112). Hillsdale, NJ: Erlbaum.

Shyan, M. R., Wright, A. A., CoOK, R. G., \& JiTSUMORI, M. (1987). Acquisition of the auditory same/different task in a rhesus monkey. Bulletin of the Psychonomic Society, 25, 1-4.

(Manuscript received January 6, 1987; revision accepted for publication November $23,1987$. 